\title{
Enhancement of Antibiotic Efficacy against Multi-drug Resistant Pseudomonas aeruginosa Infections via Combination with Curcumin and 1-(1-Naphthylmethyl)-Piperazine
}

\section{Ballard E and Coote PJ*}

Biomedical Sciences Research Complex, School of Biology, University of St Andrews, UK

\begin{abstract}
Objective: The aim of this study was to determine if the plant phenolic curcumin (CUR) and the arylpiperazine 1-(1-naphthylmethyl)-piperazine (NMP) could restore antibiotic efficacy versus MDR $P$. aeruginosa infection.

Methods: The MICs of piperacillin, meropenem and levofloxacin in the presence or absence of CUR or NMP against a MDR strain that over-expresses the MexAB-OprM efflux-pump and the isogenic parent strain were compared. The efficacy of the same combination treatments was also tested in a Galleria mellonella in vivo infection model and larval survival and bacterial burden compared.

Results: In vitro, CUR restored the activity of piperacillin, meropenem and levofloxacin versus the MDR strain of $P$. aeruginosa only weakly. There was no evidence in vitro of a similar effect with NMP. In vivo, treatment of G. mellonella larvae infected with the MDR strain with a combination of NMP or CUR plus levofloxacin, and piperacillin plus CUR, resulted in enhanced therapeutic benefit compared to the monotherapies. When compared with monotherapies, the enhanced efficacy of the combination treatments correlated with reduced bacterial burden.
\end{abstract}

Conclusion: CUR and NMP restored the efficacy of antibiotic therapy in vivo versus MDR $P$. aeruginosa infection.

Keywords: MDR Pseudomonas aeruginosa; MexAB-OprM; Eflux pump inhibitors; Curcumin; NMP; Galleria mellonella

\section{Introduction}

P. aeruginosa is an opportunistic Gram-negative pathogen accounting for significant morbidity and mortality worldwide and is the second most frequent cause of healthcare- or ventilator-associated pneumonia [1]. Multidrug resistance (MDR) is defined as resistance to 3 or more antibiotics and the incidence of MDR in P. aeruginosa is increasing [2]. As a consequence, P. aeruginosa is likely to pose a major therapeutic challenge in future [1]

Membrane-associated efflux-pumps belonging to the resistancenodulation-division (RND) family actively export antibiotics and as a consequence are major contributors to $P$. aeruginosa antibiotic resistance [1]. Of the 12-member RND family, MexAB-OprM is considered to be the most important efflux-pump mediating antibiotic resistance in $P$. aeruginosa because it transports a broad range of antibiotics [1]. Mutations in efflux-pump regulatory genes can result in efflux-pump overexpression that confers a MDR phenotype [3] and are commonly identified in clinical isolates, for example, mutations in nalB result in over-expression of MexAB-OprM, and contribute to reduced clinical efficacy of some antibiotics [4]. Treatments that combine efflux-pump inhibitors (EPIs) with antibiotics that are normally ineffective due to efflux could result in restoration of the normal clinical efficacy of the drug [5].

Curcumin (CUR) is a phenolic compound derived from the rhizomes of the plant Curcuma longa that is cultivated in India and Asia and is a constituent of the food ingredient turmeric. The compound has been studied extensively and has been shown to possess significant antimicrobial, anti-inflammatory, anti-cancer and anti-oxidant properties (reviewed in [6,7]). The antibacterial action of CUR has been attributed to inhibition of polymerisation of the essential prokaryotic cell division protein FtsZ thus preventing cytokinesis [8]. Furthermore, CUR reverses the drug resistance of cancer cells by modulating the activity of multi-drug efflux pumps [9], competitively inhibits Candida albicans ATP-binding cassette transporters and displayed synergistic inhibition of yeast strains expressing these efflux-pumps in combination with selected azoles [10], and finally, also inhibits the NorA effluxpump of Staphylococcus aureus resulting in an 8-fold reduction in the MIC of ciprofloxacin [11].

Evidence has been presented that CUR and the arylpiperazine, 1-(1-naphthylmethyl)-piperazine (NMP) could also be acting as EPIs in vitro $[12,13]$ versus Gram-negative bacteria. CUR reduced the MIC of several antibiotics against MDR isolates of $P$. aeruginosa and this was attributed to efflux-pump inhibition. Similarly, NMP was shown to reverse MDR in Escherichia coli strains over-expressing RND effluxpumps by enhancing susceptibility to antibiotics and increasing their intracellular concentration.

Employing a $P$. aeruginosa nalB-type strain that possesses mutation(s) in the MexR repressor that results in overexpression of the MexAB-OprM efflux pump [3,14], this study assessed the efficacy of combinations of antibiotics with CUR and NMP using a Galleria mellonella in vivo infection model to determine if these combinations could provide therapeutic benefit versus infections with a $P$. aeruginosa strain possessing an efflux-pump-mediated MDR phenotype.

*Corresponding author: Peter J. Coote, B.Sc, Ph.D, Biomedical Sciences Research Complex, School of Biology, University of St Andrews, The North Haugh, St Andrews Fife. KY16 9ST, UK, Tel: (44) (0)1334 463406; Fax: (44) (0)1334 462595; E-mail: pjc5@st-andrews.ac.uk

Received March 25, 2016; Accepted May 05, 2016; Published May 20, 2016

Citation: Ballard E, Coote PJ (2016) Enhancement of Antibiotic Efficacy against Multi-drug Resistant Pseudomonas Aeruginosa Infections via Combination with Curcumin and 1-(1-Naphthylmethyl)-Piperazine. J Antimicro 2: 116 doi:10.4172/2472-1212.1000116

Copyright: ( $) 2016$ Ballard E, et al. This is an open-access article distributed unde the terms of the Creative Commons Attribution License, which permits unrestricted use, distribution, and reproduction in any medium, provided the original author and source are credited. 
Citation: Ballard E, Coote PJ (2016) Enhancement of Antibiotic Efficacy against Multi-drug Resistant Pseudomonas Aeruginosa Infections via Combination with Curcumin and 1-(1-Naphthylmethyl)-Piperazine. J Antimicro 2: 116. doi:10.4172/2472-1212.1000116

\section{Materials and Methods}

\section{Bacteria and growth media}

The strains of $P$. aeruginosa used were PAO1 (PAM1020) - the isogenic parent, and PAM1032 - harbouring a mutation in nalB that results in over-expression of the RND efflux-pump MexAB-OprM [14]. The strains were a kind gift of Dr. Olga Lomovskaya, Rempex Pharmaceuticals, USA. Strains were cultured overnight in MuellerHinton Broth (MHB; Merck, Darmstadt, Germany) at $37^{\circ} \mathrm{C}$ with shaking to prepare inocula for drug susceptibility testing in vitro and efficacy testing in vivo.

Antibiotics and G. mellonella larvae. All antibiotics, Pseudomonas Isolation Agar (PIA), curcumin (CUR) ( $\geq 65 \%$ HPLC) and arylpiperazine 1-(1-naphthylmethyl)-piperazine (NMP) were purchased from SigmaAldrich Ltd (Dorset, UK). Antibiotic stock solutions were made in sterile deionised water and CUR or NMP in DMSO. Sub-stocks of all drugs for use in experiments were made in sterile deionised water. $G$. mellonella larvae were purchased from UK Waxworms Ltd (Sheffield, UK).

Antibiotic, CUR or NMP susceptibility in vitro. This was performed as previously described [15]. Briefly, the MIC of antibiotics, CUR or NMP, or combinations of CUR or NMP with antibiotics was carried out via doubling dilution of each drug in MHB and subsequent inoculation with $1.0 \times 10^{6} \mathrm{cfu} / \mathrm{mL}$ of $P$. aeruginosa PAM1020 or 1032 . For combination MICs, doubling dilutions of each antibiotic was followed by addition of CUR or NMP at three concentrations less than the MIC values for each compound. CUR and NMP were tested in combination with piperacillin (PIP), meropenem (MEM) and levofloxacin (LVX). Microplates were incubated at $37^{\circ} \mathrm{C}$ and the MIC was defined as the concentration present in the first optically clear well after $24 \mathrm{~h}$. Each experiment was performed in triplicate. Fractional inhibitory concentration index (FICI) values were calculated for each combination tested [16], and synergy was defined as FICI $\leq 0.5$. The FICI was calculated as follows: FIC of drug $\mathrm{A}=$ MIC drug A in combination/MIC of drug A alone; FIC of drug B = MIC of drug B in combination/MIC of drug B alone; thus, FICI = FIC of drug A + FIC of drug $B$.

G. mellonella model of $P$. aeruginosa infection. This was performed exactly as previously described [15]. Larvae were infected with an inoculum of $2.5 \times 10^{3} \mathrm{cfu} / \mathrm{mL}$ of $P$. aeruginosa PAM1020 or 1032 . Single doses of PIP (10, 25 and $100 \mathrm{mg} / \mathrm{kg}), \operatorname{MEM}(0.1,0.25$ and $0.5 \mathrm{mg} / \mathrm{kg})$ and $\operatorname{LVX}(0.2,1$ and $5 \mathrm{mg} / \mathrm{kg})$ were administered. A dose of $100 \mathrm{mg} /$ $\mathrm{kg}$ of CUR or $200 \mathrm{mg} / \mathrm{kg}$ of NMP was used in combination with PIP, MEM or LVX as pilot studies revealed that these doses resulted in optimal results (data not shown). Single doses of the antibiotics, CUR or NMP, or combinations, were administered $2 \mathrm{~h}$ post-infection (p.i). For triple doses of single drugs, or dual drug combinations, the second and third doses were administered 4 and $6 \mathrm{~h}$ p.i respectively. Triple doses consisted of 1 and $5 \mathrm{mg} / \mathrm{kg}$ of LVX or 10 and $100 \mathrm{mg} / \mathrm{kg}$ of PIP for PAM1020 and PAM1032 respectively. For both strains, the dose of CUR or NMP administered alone or in combination with antibiotics remained the same at $100 \mathrm{mg} / \mathrm{kg}$ or $200 \mathrm{mg} / \mathrm{kg}$ respectively.

Experimental groups contained 15 larvae and were repeated twice using larvae from different batches. Data from these replicate experiments were pooled to give $n=30$. Survival data were plotted using the Kaplan-Meier method and comparisons made between groups using the log rank test. In all comparisons to the negative control it was the uninfected control (rather than the unmanipulated control) that was used. In all tests $P \leq 0.05$ was considered significant and Holm's correction was always applied to account for multiple comparisons [17].

For haemolymph burden, groups of 30 larvae were infected with $2.5 \times 10^{3} \mathrm{cfu} / \mathrm{mL}$ of $P$. aeruginosa PAM1032. Single drug or dual combinations were administered three times at 2,4 and $6 \mathrm{~h}$ p.i. The larvae were incubated in Petri dishes at $37^{\circ} \mathrm{C}$. At $24 \mathrm{~h}$ intervals, five larvae were selected at random from each treatment group and tested for haemolymph burden exactly as previously described [15].

\section{Results}

In vitro, combination of CUR restores the antibiotic susceptibility of a P. aeruginosa strain that overexpresses the efflux-pump MexAB-OprM.

In vitro susceptibility of the parent strain (PAM1020) and the strain that overexpresses MexAB-OprM (PAM1032) to PIP, MEM and LVX, with or without the presence of CUR or NMP, is shown in Table 1. Only these antibiotics were tested because previous work with a broad range of antibiotics revealed that the greatest decreases in susceptibility occurring in the nalB-type strain compared to the parent strain (attributed to overexpression of MexAB-OprM) occurred with these drugs [15]. Confirming earlier work [15], nalB-dependent overexpression of MexAB-OprM conferred resistance to PIP, MEM and LVX compared to the isogenic parent (PAM1020).

NMP alone had no significant inhibitory activity against either of the $P$. aeruginosa strains tested: the MIC for both PAM1020 and 1032 was $>128 \mathrm{mg} / \mathrm{L}$. CUR alone had weak inhibitory activity with a MIC of $64 \mathrm{mg} / \mathrm{L}$ for both strains. For the parent strain (PAM1020), combination of NMP with any of the three antibiotics tested did not reduce their MIC values but combination with CUR reduced the antibiotic MICs slightly. However, the calculated FICI values for all of these combinations versus PAM1020 indicated no significant synergistic inhibition $(>0.5)$. A similar pattern of results were observed for the combination of NMP or CUR with the same antibiotics versus the MDR strain (PAM1032). With this strain, combination of CUR with each of the three antibiotics did reduce the antibiotic MIC values to a greater extent than with the parent strain and this was reflected in lower FICI values. Nevertheless, the FICI values were still greater than 0.5 indicating that the interaction between CUR and the antibiotics was not strongly synergistic.

The effect of combination of CUR with PIP, MEM and LVX on the viability of PAM1020 and PAM1032 after 24 h exposure is shown in Table 2. Data for NMP is not shown because no differences in viability were detected for either strain for any of the drug combinations tested. Supporting the data in Table 1, combination of CUR with the antibiotics did reduce the viability of the MDR strain, PAM1032, by almost $1 \log _{10}$ $\mathrm{cfu} / \mathrm{mL}$ compared to the individual treatments. The same combinations had little additional effect over and above the individual treatments on the viability of the parent strain PAM1020.

In conclusion, CUR had a weak restorative effect on the activity of PIP, MEM and LVX versus a $P$. aeruginosa strain with an efflux-pumpmediated MDR phenotype in vitro. There was no evidence in vitro of a similar effect with NMP.

In G. mellonella larvae, combination of CUR or NMP with antibiotics results in enhanced efficacy versus MDR P. aeruginosa infection.

The same combinations were then investigated for in vivo efficacy in G. mellonella larvae. Importantly, neither CUR nor NMP, when administered alone at the highest doses tested, 100 and $200 \mathrm{mg} / \mathrm{kg}$ 
Citation: Ballard E, Coote PJ (2016) Enhancement of Antibiotic Efficacy against Multi-drug Resistant Pseudomonas Aeruginosa Infections via Combination with Curcumin and 1-(1-Naphthylmethyl)-Piperazine. J Antimicro 2: 116. doi:10.4172/2472-1212.1000116

Page 3 of 6

\begin{tabular}{|c|c|c|c|c|c|c|}
\hline \multirow{2}{*}{ Strain } & \multirow{2}{*}{ Drug } & \multicolumn{3}{|c|}{ Drug MIC (mg/L) with } & \multicolumn{2}{|c|}{$\mathrm{FICl}^{2}$} \\
\hline & & No EPI & NMP $^{1}$ & CUR $^{1}$ & Drug + NMP & Drug + CUR \\
\hline & PIP & $4-8$ & 4 & 2 & 1.78 & 1.28 \\
\hline \multirow[t]{3}{*}{ PAM1020 } & MEM & $0.5-1$ & 1 & 0.5 & 2.78 & 1.78 \\
\hline & LVX & 0.5 & 0.5 & 0.25 & 1.78 & 1.28 \\
\hline & PIP & $16-32$ & 16 & 2 & 1.78 & 0.96 \\
\hline \multirow[t]{2}{*}{ PAM1032 } & MEM & 4 & 4 & 0.125 & 1.78 & 0.81 \\
\hline & LVX & 2 & 2 & 0.03 & 1.78 & 0.79 \\
\hline
\end{tabular}

${ }^{1}$ The MIC versus both PAM 1020 and 1032 was $>128 \mathrm{mg} / \mathrm{L}$ for NMP and $64 \mathrm{mg} / \mathrm{L}$ for CUR. The concentration of NMP or CUR added to each well was selected to be lower than the MIC for each strain: NMP $(1,10$ or $100 \mathrm{mg} / \mathrm{L})$ and CUR $(0.5,5$ or $50 \mathrm{mg} / \mathrm{L})$ and the FICl value was calculated using the highest concentration tested.

${ }^{2}$ Fractional inhibitory concentration index (FIC index) where synergistic $(\leq 0.5)$, non-synergistic $(>0.5-\leq 2.0)$ and antagonistic $(>4)$. An actual MIC was not determined for NMP so the highest value tested was used in the $\mathrm{FICl}$ calculation to provide a conservative estimate of the $\mathrm{FICl}$ value.

Table 1: Minimum inhibitory concentrations (MICs) of three antibiotics in the presence or absence of NMP or CUR against $P$ seudomonas aeruginosa PAM1020 and 1032 Each experiment was completed at least in duplicate.

\begin{tabular}{|c|c|c|c|c|c|c|}
\hline Strain & Treatment & Concentration & Inoculum & Untreated control & Treatment ( \pm SD) & Log reduction \\
\hline & & (mg/L) & \multirow{8}{*}{$6.71 \pm 0.137$} & \multirow{8}{*}{$9.85 \pm 0.008$} & & \\
\hline \multirow{7}{*}{ PAM1020 } & PIP & 0.5 & & & $9.72 \pm 0.02$ & 0.13 \\
\hline & MEM & 0.0625 & & & $9.69 \pm 0.01$ & 0.16 \\
\hline & LVX & 0.125 & & & $9.69 \pm 0.04$ & 0.16 \\
\hline & CUR & 32 & & & $9.69 \pm 0.05$ & 0.16 \\
\hline & PIP + CUR & $0.5+32$ & & & $9.63 \pm 0.05$ & 0.22 \\
\hline & MEM + CUR & $0.0625+32$ & & & $9.67 \pm 0.02$ & 0.18 \\
\hline & LVX + CUR & $0.25+32$ & & & $9.63 \pm 0.04$ & 0.22 \\
\hline \multirow{7}{*}{ PAM1032 } & PIP & 4 & \multirow{7}{*}{$6.72 \pm 0.14$} & \multirow{7}{*}{$9.69 \pm 0.029$} & $9.83 \pm 0.02$ & -0.14 \\
\hline & MEM & 1 & & & $9.69 \pm 0.12$ & 0 \\
\hline & LVX & 2 & & & $9.70 \pm 0.13$ & -0.01 \\
\hline & CUR & 32 & & & $9.85 \pm 0.12$ & -0.16 \\
\hline & PIP + CUR & $4+32$ & & & $8.83 \pm 0.02$ & 0.86 \\
\hline & MEM + CUR & $1+32$ & & & $8.82 \pm 0.06$ & 0.87 \\
\hline & LVX + CUR & $2+32$ & & & $8.79 \pm 0.07$ & 0.9 \\
\hline
\end{tabular}

Table 2: The effect of dual combinations of antibiotics with curcumin on the viability of $P$. aeruginosa PAM1020 and PAM1032 in vitro. Viability was determined in 96-well microplates after $24 \mathrm{~h}$ exposure to the antibiotics in MHB at $37^{\circ} \mathrm{C}$. Data shown is the mean and standard deviation.

respectively, had any toxic effect on the larvae (data not shown). Initial experiments compared the efficacy of a single dose administered $2 \mathrm{~h}$ post-infection (p.i) of the antibiotics alone (PIP, MEM or LVX), the EPIs alone (CUR or NMP) and each antibiotic in combination with CUR or NMP. Treatment with the combinations NMP + PIP, CUR + MEM and NMP + MEM conferred no therapeutic benefit compared to antibiotic monotherapy alone. The combinations of NMP + LVX and CUR + PIP or LVX resulted in a small increase in survival after $96 \mathrm{~h}$, compared to PBS or monotherapies, of G. mellonella larvae infected with the MDR strain of $P$. aeruginosa (PAM1032) (data not shown). None of the combination treatments conferred any additional therapeutic benefit over monotherapies upon larvae infected with the parent strain PAM1020 (data not shown).

The three combination treatments that demonstrated some enhanced therapeutic benefit after a single dose were then tested using a triple dose administered 2, 4 and $6 \mathrm{~h}$ p.i (Figure 1). A triple dose of LVX + NMP resulted in a significant increase in survival compared to triple doses of any of the monotherapies over $96 \mathrm{~h}(P \leq 0.05)$. This enhanced therapeutic benefit of the combination treatment was observed for both the parent strain (PAM1020) and the MDR strain (PAM1032). Notably, this result contradicts the results seen in vitro where the combination of LVX with NMP was not observed to be synergistic versus either strain (Table 1).

Triple doses of PIP + CUR and LVX + CUR also induced significantly enhanced survival over their constituent monotherapies $(P$ $\leq 0.05$ ) but this was only observed against infection caused by the MDR strain. In contrast to the data obtained with NMP, the enhancement of antibiotic efficacy observed with CUR was partly supported by the in vitro data that showed CUR increased susceptibility of PAM1032 to all three antibiotics tested (Table 1).

The effect of triple doses of the two most efficacious combination therapies LVX + NMP and PIP + CUR on the larval burden of the MDR strain (PAM1032) was compared (Figure 2a and 2b). Larval burden following three doses of either NMP (Figure 2a) or CUR (Figure 2b) alone increased from below the level of detection $\left(\leq 2 \log _{10} \mathrm{cfu} / \mathrm{mL}\right) 5$ $\mathrm{h}$ post-infection to greater than $8 \log _{10} \mathrm{cfu} / \mathrm{mL}$ after $24 \mathrm{~h}$ at $37^{\circ} \mathrm{C}$. This rapid proliferation of $P$. aeruginosa seen $24 \mathrm{~h}$ after infection correlated with death of the larval population (Figure 1). Treatment with three doses of LVX or PIP alone did retard bacterial growth over $96 \mathrm{~h}$ which in both cases was reflected by some larval survival. Notably, treatment with triple doses of LVX + NMP or PIP + CUR reduced larval burden compared to the monotherapies reflecting the higher levels of larval survival observed (Figure 1).

In summary, combination of antibiotics with CUR or NMP can restore antibiotic efficacy in vivo versus infections with a strain of $P$. aeruginosa possessing an efflux-pump-mediated MDR phenotype and this enhanced efficacy of the combination treatments correlates with reduced larval burden of the infecting pathogen. Importantly, this therapeutic benefit was not detected in comparative in vitro studies. 
Citation: Ballard E, Coote PJ (2016) Enhancement of Antibiotic Efficacy against Multi-drug Resistant Pseudomonas Aeruginosa Infections via Combination with Curcumin and 1-(1-Naphthylmethyl)-Piperazine. J Antimicro 2: 116. doi:10.4172/2472-1212.1000116

PAM1020
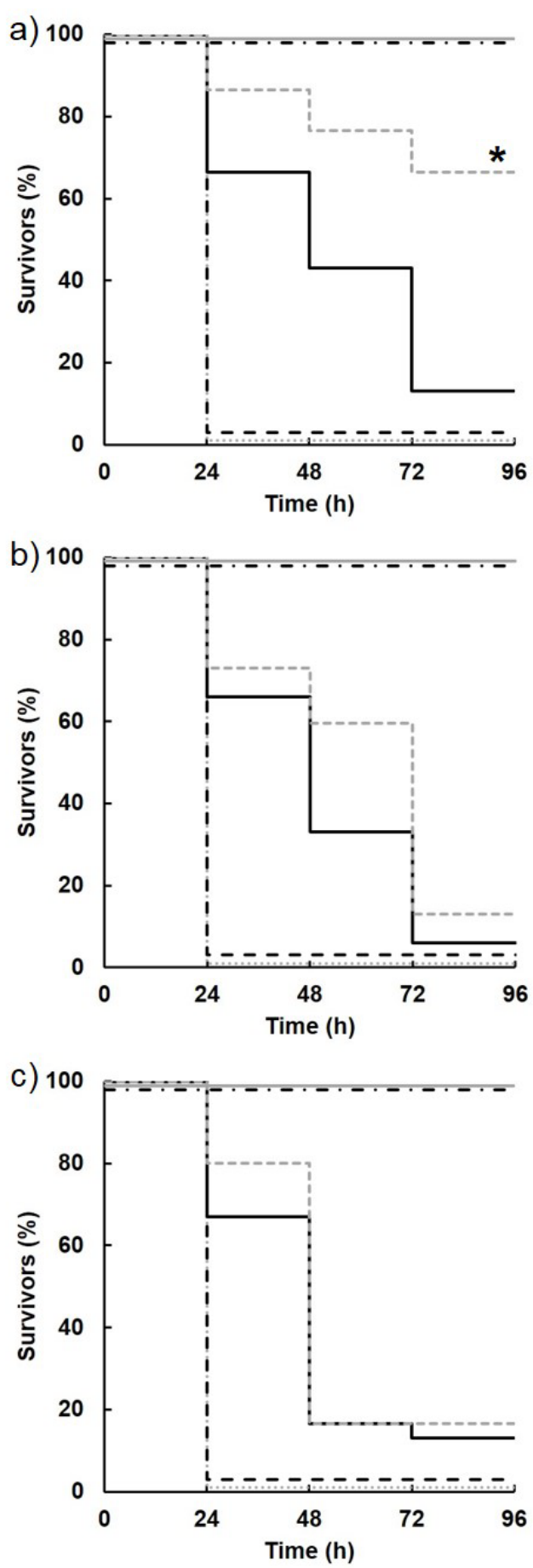

PAM1032
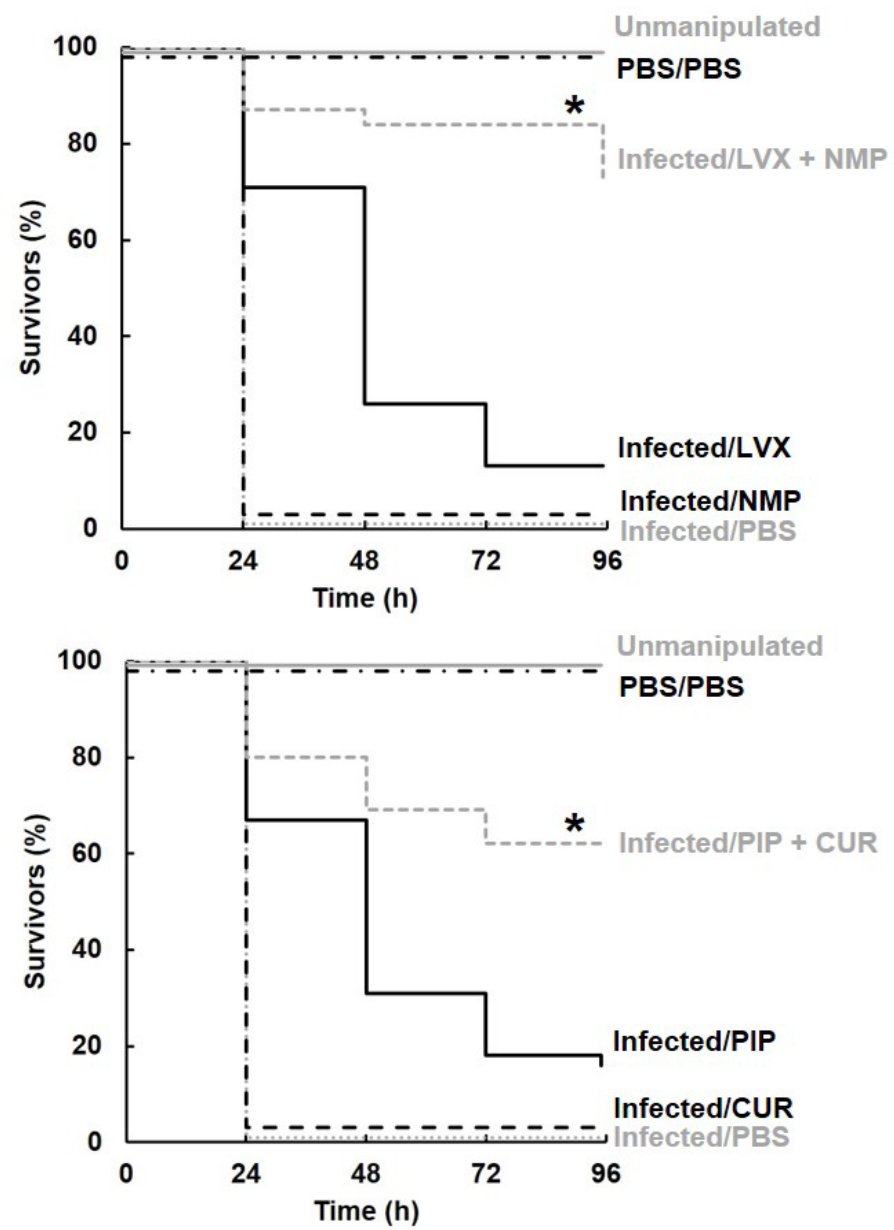

Unmanipulated

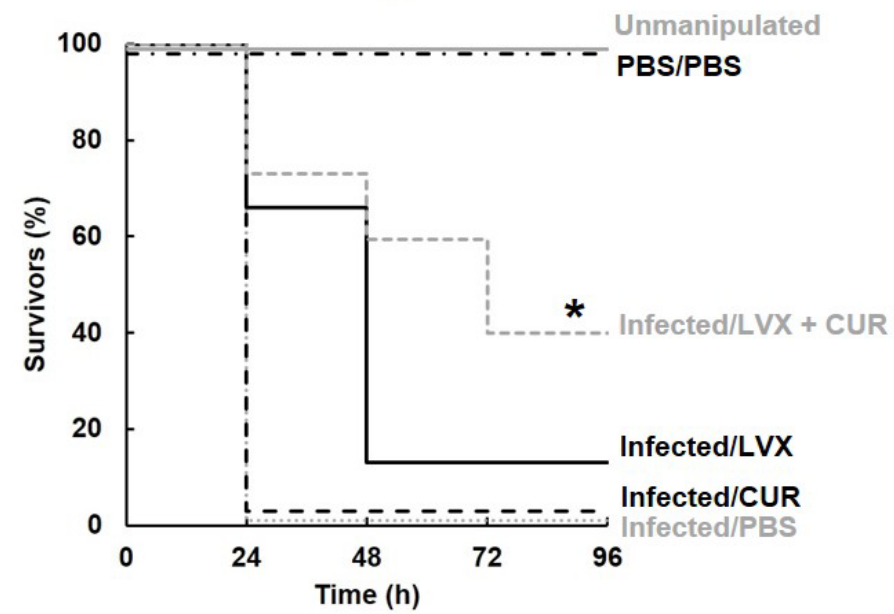

Figure 1: Effect of treatment with combinations of (a) LVX + NMP, (b) PIP + CUR and (c) LVX + CUR on survival of G. mellonella larvae infected with P. aeruginosa PAM1020 or PAM1032. All larvae were inoculated with $2.5 \times 10^{3} \mathrm{cfu} / \mathrm{mL} P$. aeruginosa and treated with each drug individually or in combination with three doses at 2, 4 and $6 \mathrm{~h}$ p.i. Treatments consisted of PBS, NMP (200 mg/kg), CUR (100 mg/kg), LVX (PAM1020 $1 \mathrm{mg} / \mathrm{kg} ;$ PAM1032 $5 \mathrm{mg} / \mathrm{kg})$ and PIP (PAM1020 10 mg/kg; PAM1032 $100 \mathrm{mg} / \mathrm{kg}$ ) alone, or combinations of the antibiotics with CUR or NMP administered at the same dosages. Larvae were incubated at $37^{\circ} \mathrm{C}$ for $96 \mathrm{~h}$ and survival recorded every $24 \mathrm{~h} .{ }^{*}$ indicates a combination treatment group with significantly enhanced survival compared to any of the constituent monotherapies $(P$ $<0.05$, log-rank test with Holm correction for multiple comparisons). $n=30$ (pooled from duplicate experiments). 

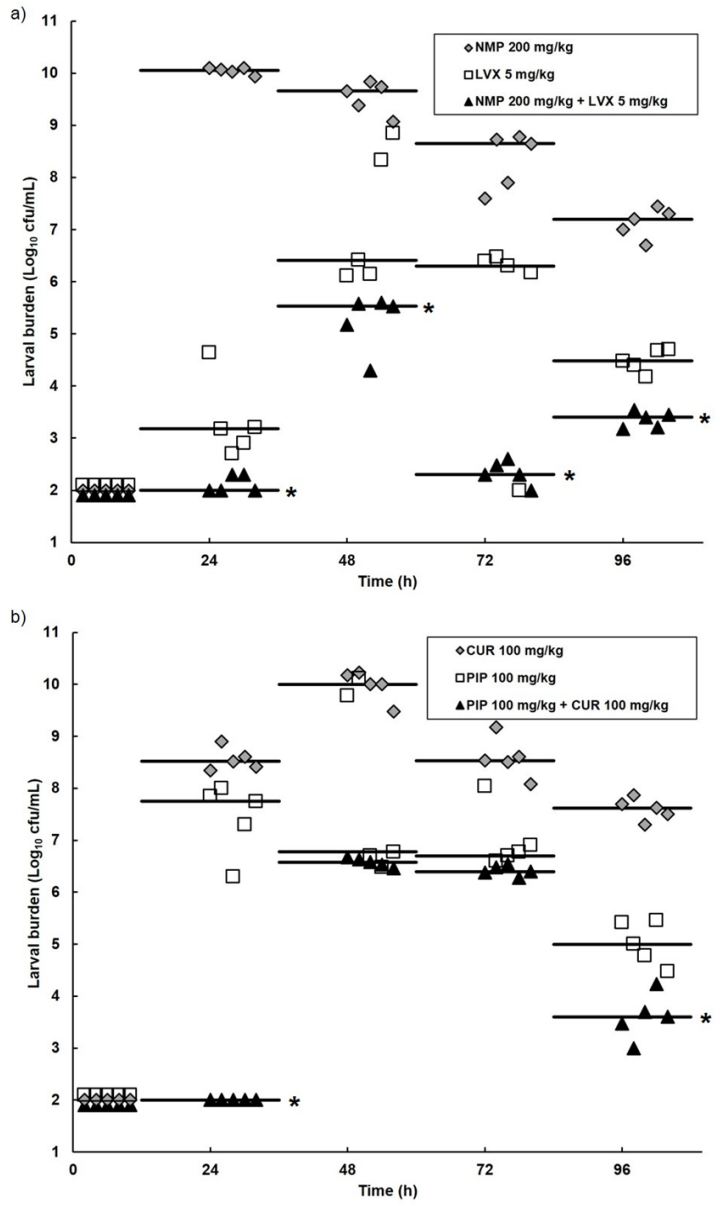

Figure 2: Effect of treatment with combinations of (a) LVX + NMP and (b) PIP + CUR on larval burden of $P$. aeruginosa PAM1032. All larvae were inoculated with $2.5 \times 10^{3} \mathrm{cfu} / \mathrm{mL} P$. aeruginosa and treated with three doses of each individual drug or combinations at 2, 4 and $6 \mathrm{~h}$ p.i. Treatments consisted of PBS, LVX (5 mg/kg), PIP (100 mg/kg), NMP (200 mg/kg) or CUR (100 mg/ $\mathrm{kg}$ ) alone, or combinations of the antibiotics with CUR or NMP administered at the same dosages. Larvae were incubated at $37^{\circ} \mathrm{C}$ for $96 \mathrm{~h}$ and the burden of $P$ aeruginosa determined from 5 individual larvae every $24 \mathrm{~h}$. For clarity, data for treatment with PBS alone is not shown because the data obtained was similar to that obtained for NMP or CUR treatment alone. * indicates a significant difference in larval burden between groups treated with the combination therapies compared to the constituent monotherapies; $n=5(P<0.05$, unpaired $\mathrm{t}$-test). The black bar represents the median value of larval burden per group.

\section{Discussion}

Many antibiotic-resistant clinical isolates of $P$. aeruginosa that prove difficult to treat overexpress efflux-pumps [3]. The use of EPIs in combination treatments with existing antibiotics have been proposed as a potential solution to the lack of new antibiotics suitable for treating MDR $P$. aeruginosa infections that would restore the efficacy of drugs normally rendered ineffective by efflux-pumps [5].

CUR possesses antimicrobial properties but has only weak activity versus $P$. aeruginosa [12]. This work confirmed this weak inhibitory activity. CUR attenuated virulence of $P$. aeruginosa in a Caenorhabditis elegans infection model which was attributed to down-regulation of quorum-sensing, virulence factor production and biofilm formation [18].
Notably, a recent study showed that CUR reduced the antibiotic MIC of MDR clinical isolates of $P$. aeruginosa in vitro and this was attributed to efflux-pump inhibition despite no specific evidence for this being presented [12]. Supporting this, the in vitro data presented here showed that CUR reversed the antibiotic resistance of an MDR P. aeruginosa isolate but only weakly. In vitro, CUR had a greater inhibitory effect in combination with the antibiotics on PAM1032 than the parent strain PAM1020. Thus, it cannot be discounted that this enhanced inhibitory effect of CUR with antibiotics in the strain with the nalB background could be due to other effects of this mutation that are independent of the over-expression of the MexAB-OprM efflux pump.

Bohnert et al. [13] reported that the arylpiperazine NMP was able to reverse $E$. coli antibiotic resistance in vitro due to efflux-pump inhibition. Subsequently, NMP was shown to act via competitive inhibition of efflux-pumps [19]. However, in this work NMP did not reverse the efflux-pump mediated resistance of $P$. aeruginosa to PIP, MEM or LVX in vitro. Supporting this, a recent study revealed that NMP did not reduce the ciprofloxacin MIC of a series of ciprofloxacin-resistant $P$. aeruginosa clinical isolates in contrast to another EPI, phenylalanine-arginine- $\beta$-naphthylamide [20].

Using G. mellonella larvae, the present study has shown that combinations of NMP with LVX and CUR with PIP or LVX conferred significant therapeutic benefit compared to the individual monotherapies in vivo to larvae infected with a MDR strain of $P$. aeruginosa. In each example, CUR or NMP was able to restore the efficacy of each of the administered antibiotics to varying degrees. These results support the aforementioned studies showing that the co-administration of antibiotics with CUR or NMP reverses MDR phenotypes in vitro but does not prove that this is due to efflux-pump inhibition.

Notably, there were discrepancies between the results observed in vitro with those in vivo - for example, NMP + LVX, the most potent combination in vivo was not synergistic in vitro, and CUR + MEM had one of the lowest FICI scores in vitro but conferred no therapeutic benefit in vivo. Similar discrepancies were also seen in a previous study that employed the G. mellonella infection model [15]. There are many examples where combinations of antibiotics are shown to have enhanced activity in vitro but offer no therapeutic benefit when tested in vivo (reviewed in [21]). Thus, screening for novel antibiotic treatments in vitro is not a good predictor of in vivo efficacy and instead should be performed in rapid, tractable in vivo infection models such as G. melonella larvae to ensure a greater chance of success when these treatments are tested in subsequent mammalian infection models.

Both CUR and NMP have poor aqueous solubility which is not ideal for a prospective drug candidate. However, in an attempt to improve oral bioavailability of CUR researchers have investigated coupling the compound to nanocarriers. Thus, curcumin-loaded poly lactideco-glycolide and curcumin nanoparticles had superior bioactivity and bioavailability in addition to enhanced cellular uptake compared to curcumin alone [22]. In addition, encapsulation of curcumin with gelatin or starch improved the solubility and stability of the compound whilst retaining antimicrobial activity [23].

Future work will be required to confirm the enhanced efficacy of these combination treatments in mammalian infection models and to determine if either CUR or NMP has potential for clinical application.

\section{Acknowledgements}

This work was funded by the University of St Andrews. 
Citation: Ballard E, Coote PJ (2016) Enhancement of Antibiotic Efficacy against Multi-drug Resistant Pseudomonas Aeruginosa Infections via Combination with Curcumin and 1-(1-Naphthylmethyl)-Piperazine. J Antimicro 2: 116. doi:10.4172/2472-1212.1000116

\section{References}

1. Poole K (2011) Pseudomonas aeruginosa: resistance to the max. Front Microbiol 2: 1-13.

2. Hirsch EB, Tam VH (2010) Impact of multidrug-resistant Pseudomonas aeruginosa infection on patient outcomes. Exp Rev Pharmacoecon Outcomes Res 10: 441-451.

3. Lister PD, Wolter D, Hanson N (2009) Antibacterial-resistant Pseudomonas aeruginosa: clinical impact and complex regulation of chromosomally encoded resistance mechanisms. Clin Microbiol Rev 22: 582-610.

4. Chen HY, Yuan M, Livermore DM (1995) Mechanisms of resistance to betalactam antibiotics amongst Pseudomonas aeruginosa isolates collected in the UK in 1993. J Med Microbiol 43: 300-309.

5. Kourtesi C, Ball AR, Huang YY, Jachak SM, Vera DMA, et al. (2013) Microbial efflux systems and inhibitors: approaches to drug discovery and the challenge of clinical implementation. The Open Microbiol J 7: 34-52.

6. Moghadamtousi SZ, Kadir HA, Hassandarvish P, Tajik H, Abubakar S, et al. 2014) A review on antibacterial, antiviral, and antifungal activity of curcumin BioMed Res Int.

7. Epstein J, Sanderson IR, MacDonald TT (2009) Curcumin as a therapeutic agent: the evidence from in vitro, animal and human studies. Brit J Nutrit 103: $1545-1557$.

8. Rai D, Singh JK, Roy N, Panda D (2008) Curcumin inhibits FtsZ assembly: an attractive mechanism for its antibacterial activity. Biochem J 410: 147-155.

9. Chearwae W, Shukla S, Limtrakul P, Ambudkar SV (2006) Modulation of the function of the multidrug resistance-linked ATP-binding cassette transporter ABCG2 by the cancer chemopreventive agent curcumin. Mol Cancer Ther 5 : 1995-2006.

10. Sharma M, Manoharlal R, Shukla S, Puri N, Prasad T, et al. (2009) Curcumin modulates efflux mediated by yeast $A B C$ multidrug transporters and is synergistic with antifungals. Antimic Agents Chemother 53: 3256-3265

11. Joshi P, Singh S, Wani A, Sharma S, Jain SK, et al. (2014) Osthol and curcumin as inhibitors of human Pgp and multidrug efflux pumps of Staphylococcus aureus: reversing the resistance against frontline antibacterial drugs. Med Chem Commun 5: 1540-1547.

12. Negi N, Prakash P, Gupta ML, Mohapatra TM (2014) Possible role of curcumin as an efflux pump inhibitor in multi drug resistant clinical isolates of Pseudomonas aeruginosa. J Clin Diag Res 8: 4-7.

13. Bohnert JA, Kern WV (2005) Selected arylpiperazines are capable of reversing multidrug resistance in Escherichia coli overexpressing RND efflux pumps. Antimicrob Agents Chemother 49: 849-852.

14. Lomovskaya O, Lee A, Hoshino K, Ishida H, Mistry A, et al. (1999) Use of a genetic approach to evaluate the consequences of inhibition of efflux pumps in Pseudomonas aeruginosa. Antimicrob Agents Chemother 43: 1340-1346.

15. Adamson DH, Krikstopaityte V, Coote PJ (2015) Enhanced efficacy of putative efflux pump inhibitor/antibiotic combination treatments versus MDR strains of Pseudomonas aeruginosa in a Galleria mellonella in vivo infection model. J Antimic Chemother 70: 2271-2278.

16. Climo MW, Patron RL, Archer GL (1999) Combinations of vancomycin and beta-lactams are synergistic against staphylococci with reduced susceptibilities to vancomycin. Antimic Agents Chemother 43: 1747-1753.

17. Holm S (1979) A simple sequentially rejective multiple test procedure. Scand J Stat 6: 65-70.

18. Rudrappa T, Bais HP (2008) Curcumin, a known phenolic from Curcuma longa attenuates the virulence of Pseudomonas aeruginosa PAO1 in whole plant and animal pathogenicity models. J Agric Food Chem 56: 1955-1962.

19. Bohnert JA, Karamian B, Nikaido H (2010) Optimized Nile Red efflux assay of AcrAB-TolC multidrug efflux system shoes competition between substrates. Antimicrob Agents Chemother 54: 3770-3775.

20. Sonnet P, Izard D, Mullié C (2012) Prevalence of efflux-mediated ciprofloxacin and levofloxacin resistance in recent clinical isolates of Pseudomonas aeruginosa and its reversal by the efflux pump inhibitors 1-(1-naphthylmethyl)piperazine and phenylalanine-arginine- $\beta$-naphthylamide. Int $\mathrm{J}$ Antimicrob Agents 39: $77-80$.

21. Tamma PD, Cosgrove SE, Maragakis LL (2012) Combination therapy fo treatment of infections with Gram-negative bacteria. Clin Microbiol Rev 25 : 450-470.

22. Anand $P$, Nair HB, Sung B, Kunnumakara AB, Yadav VR, et al. (2010) Design of curcumin-loaded nanoparticles formulation with enhanced cellular uptake and increased bioactivity in vitro and superior bioavailability in vivo. Biochem Pharmacol 79: 330-338.

23. Wang Y, Lu Z, Wu H, Lv F (2009) Study on the antibiotic activity of microcapsule curcumin against foodborne pathogens. Int J Food Microbiol 136: 71-74. 